MARIA IZABEL COSTA

\title{
POLÍTICA DE DESIGN PARA O FOMENTO DA NOVAÇÃO NA CADEIA DE VALOR TÊXTIL/ CONFECÇÃO DE MODA DE SANTA CATARINA
}

Tese de Doutorado

Tese apresentada ao Programa de Pósgraduação em Design da PUC-RIO como parte dos requisitos parciais para obtenção do título de Doutora em Design.

Orientador: Cláudio Freitas de Magalhães 


\title{
POLÍTICA DE DESIGN PARA O FOMENTO DA INOVAÇÃO NA CADEIA DE VALOR TÊXTIL/CONFECÇÃO DE MODA DE SANTA CATARINA
}

\begin{abstract}
Tese apresentada como requisito parcial para obtenção do grau de Doutor pelo Programa de Pós-graduação em Design do Departamento de Artes \& Design do Centro de Teologia e Ciências Humanas da PUC-Rio. Aprovada pela Comissão Examinadora abaixo assinada.
\end{abstract}

Prof. Dr. Cláudio Freitas de Magalhães Orientador Departamento de Artes \& Design - PUC-Rio

Prof. Dr. Domingos Manfredi Naveiro Instituto Nacional de Teconologia - INT

Profa. Dra. Evelise Anicet Rüthschilling Universidade Federal do Rio Grande do Sul - UFRGS

Profa. Dra. Izabel Maria de Oliveira Departamento de Artes \& Design - PUC-Rio

Prof. Dr. Alfredo Jefferson de Oliveira Departamento de Artes \& Design - PUC-Rio

Profa. Dra. Denise B. Portinari

Coordenadora Setorial do Centro de Teologia e Ciências Humanas PUC-Rio 
Todos os direitos reservados. É proibida a reprodução total ou parcial do trabalho sem autorização da universidade, da autora e do orientador.

\section{MARIA IZABEL COSTA}

Professora do Curso de Bacharelado em Moda do Centro de Artes - UDESC Universidade do Estado de Santa Catarina UDESC/CEART.Especialista em Desenho Industrial, Estilismo e Modelagem de Moda - UDESC. Mestre em Engenharia de Produção na Área de Gestão do Design UFSC. Doutoranda em Design - Departamento de Artes e Design, Pontifícia Universidade Católica do Rio de Janeiro PUC-RIO.

Ficha Catalográfica

COSTA, Maria Izabel

Política de design para o fomento da inovação na cadeia de valor têxtil/confecção de moda./ Maria Izabel Costa; orientador: Cláudio Freitas de Magalhães. - Rio de Janeiro: PUC, Departamento de Artes e Design,2011.

v., f.:270 il. ; $29,7 \mathrm{~cm}$.

1. Tese (doutorado) - Pontifícia Universidade Católica do Rio de Janeiro, Departamento de Artes e Design.

Inclui referências bibliográficas 
Ao meu pai Aroldo e minha mãe Alba Lygia, por estarem sempre ao meu lado, e aos meus filhos, Fabio e Elisa Bell, que transbordam de amor o meu coração. 


\section{Agradecimentos}

Contei com a inestimável ajuda de muitas pessoas. Cada uma, com sua colaboração específica. Juntas, deram-me o aporte para que eu pudesse realizar este trabalho com dedicação e serenidade.

Em primeiro lugar, agradeço ao Prof. Dr. Cláudio Freitas de Magalhães, meu orientador, pelas reflexões, apoio, confiança, valiosas sugestões e acertados questionamentos. Hoje, é mais que um orientador, é meu amigo.

Aos professores do Programa de Pós-graduação da PUC-Rio pelos conhecimentos, exemplos, incentivo e amizade.

Aos membros das bancas que contribuíram significativamente para o aperfeiçoamento deste trabalho. 
Aos colegas de doutorado e meus grandes amigos do Departamento de Moda da UDESC, em especial a professora Icléia Silveira, que é amiga de todas as horas, e a professora Lurdes Pulls, pelo carinho e amizade .

Sou muito grata a todas as empresas e instituições de ensino do Programa Santa Catarina Moda Contemporânea - SCMC, que participaram do processo de pesquisa. Em especial, a todas as pessoas destas organizações, e não são poucas, que abriram portas, trocaram ideias, levantaram questionamentos, me apoiaram e criaram junto comigo novos conhecimentos. Estas atitudes e ações foram fundamentais para o enriquecimento e realismo deste estudo.

\footnotetext{
À Universidade do Estado de Santa Catarina - UDESC e à Pontifícia Universidade Católica do Rio de Janeiro - PUC - RIO, pela oportunidade concedida para a realização do Doutorado em Design.
}

Por fim, agradeço o apoio de meus queridos filhos e da minha mãe, que muito sabiamente, sempre me têm ajudado com muito empenho e amor. 


\section{Resumo}

Costa, Maria Izabel. Política de design para o fomento da inovação na cadeia de valor têxtil/confecção de Santa Catarina. Rio de janeiro, 2011. 270p. Tese de doutorado - Departamento de Artes e Design. Pontifícia Universidade católica do Rio de janeiro.

Esta tese apresenta uma sistemática participativa para propor políticas de design convergentes entre universidade e empresa, para o fomento da inovação do setor têxtil catarinense. Caracteriza-se como uma pesquisa-ação de caráter quantitativo e qualitativo. Investiga empresas do setor têxtil/confecção e instituições de ensino, participantes do projeto Santa Catarina Moda Contemporânea - SCMC, com relação à inovação, utilização do design e características da relação Universidade-Empresa. Os resultados indicam que há uma demanda de inovação e design. As empresas são inovadoras, desenvolvem atividades inovativas, mas o grau de novidade é baixo. A inovação dá-se principalmente por ações da própria empresa, estando as universidades ainda à margem desse processo. Embora recente, o design de moda está presente na maioria das empresas pesquisadas, que são de médio e grande porte. A pesquisa revelou, também, que os entraves, encontrados na relação Universidade-Empresa, podem estar associados tanto à dimensão da infraestrutura destas, quanto à dimensão psicossociológica e organizacional do próprio relacionamento. Estes dados foram trabalhados em um fórum, com a participação dos 
atores envolvidos e profissionais da moda. Neste evento, foram identificados, entre outros, os principais atributos de valor da cadeia têxtil/confecção de moda catarinense que devem ser explorados na política de design têxtil: ações cooperativas que exercitam a identidade local; fortalecimento da relação universidade/empresa/governo; criação de laboratórios experimentais; promoção do design local e gestão do conhecimento. O conjunto de elementos da pesquisa-ação possibilitou a formulação de indicadores teóricos, estrutural, estratégico e instrumental que podem nortear a política de design para a inovação. Conclui-se que a sistemática proposta, de definição participativa e convergente entre empresas e universidades, favorece o desenvolvimento de políticas de design para a inovação mais direcionadas às necessidades e potencialidades de uma região específica. Possibilita às pessoas serem ativas nas decisões que lhes dizem respeito, o que as leva a serem mais propensas a adotar novas formas e condutas à inovação.

\section{Palavras-chave:}

Moda; Política de Design; Integração Universidade-Empresa; Cadeia Têxtil; Inovação. 


\section{Abstract}

Costa, Maria Izabel. Magalhães, Claudio Freitas (advisor) Design policy to foster innovation in the value chain textile / fashion business in Santa Catarina. Rio de janeiro, 2011.270p. Ph.D. thesis - Department of Art and Design. Pontifícia Universidade Católica do Rio de Janeiro.

This thesis presents a systematic participatory to propose convergent design policies between university and company to foster innovation in the textile industry of Santa Catarina. It is characterized as an action research of quantitative and qualitative feature. It looks into companies in the textile / clothing department and educational institutions, the project participants of Santa Catarina Contemporary Fashion - SCMC, concerning innovation, use of design and features of the relationship university -company. The results indicate that there is a requirement for innovation and design. The companies are innovative in that they develop innovation activities, but novelty is low. Innovation takes place mainly by actions of the company itself, while the universities are still outside of this process. Although recent, fashion design is present in most of the surveyed companies, which are medium and large sized. The survey also revealed that the drawbacks found in the relationship university - company, may be associated with both the proportion of their infrastructure and the organizational plus psychosocial extent of their own relationship. These data were worked in a forum with the participation of actors and professionals involved in fashion. In this event, the key attributes of value of the textile / fashion chain business in Santa Catarina have been identified, 
among others, which must be explored in the policy of textile design: cooperative actions that exercise the local identity, strengthening of the relationship university / company / government; creation of experimental laboratories; promoting of the local design and the knowledge management. The set of elements of action research enabled the formulation of theoretical, structural, strategic and instrumental indicators that can guide policy design for innovation. We conclude that the systematic proposal, participatory and convergent setting between companies and universities, encourages the development of policies for design innovation directed towards the needs and potentials of a specific region .It enables people to be active in decisions that concern them, which leads them to be more likely to adopt new kinds of behavior and ways to innovation.

\section{Keywords:}

Fashion; Design Policy; University-Enterprise Integration; Textile Chain; Innovation. 


\section{Sumário}

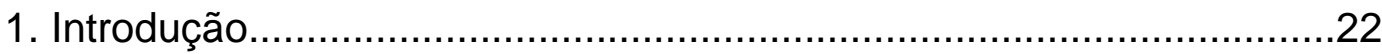

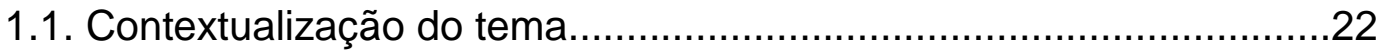

1.2. Problema de pesquisa.......................................................26

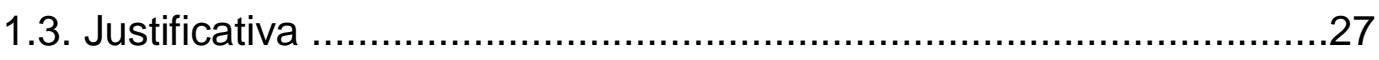

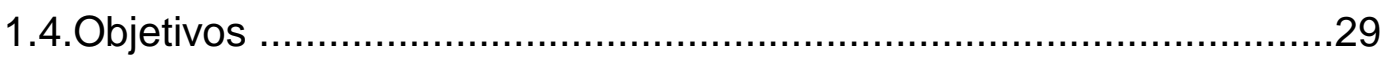

1.4.1. Objetivo geral...............................................................29

1.4.2. Objetivo específico ......................................................29

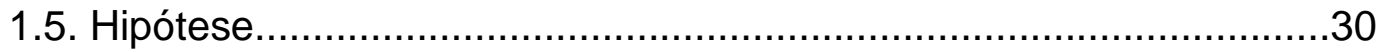

1.6. Procedimentos metodológicos ..................................................30

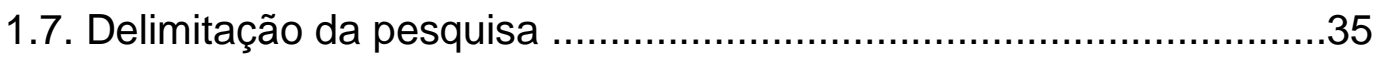

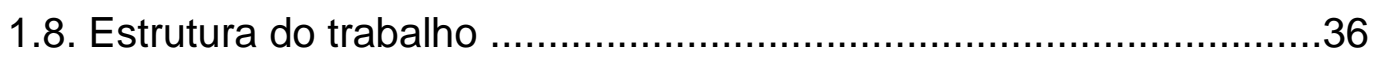

2. A Cadeia de Valor Têxtil/Confecção de Moda...................................38

2.1. Cadeia de Valor Têxtil/Confecção de Moda: estrutura

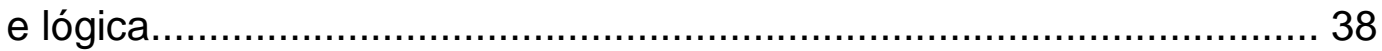

2.2. A formação das cadeias globais de produção da Indústria têxtil/confecção .......................................................... 42

2.3. Competitividade do setor produtivo têxtil/confecção no Brasil......................................................................... 48

2.4. A indústria têxtil catarinense: adaptação a um novo ambiente

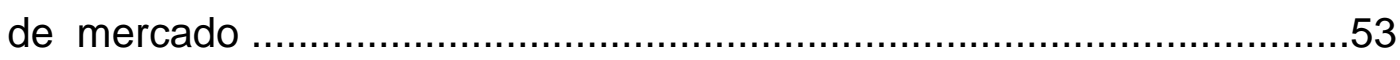

2.5. Síntese e conclusões ........................................................59

3. Inovação, design e moda .....................................................61

3.1. Conceitos e abordagens construtivas à inovação ...........................61

3.1.1. Abordagem econômica .........................................................65

3.1.2. Abordagem da difusão do conhecimento e tecnologia ...................65

3.1.3. Abordagem da organização industrial ......................................67

3.1.4. Abordagem da área da gestão .................................................72 
3.2. Tipos de inovação e sua relação com a inovação na área

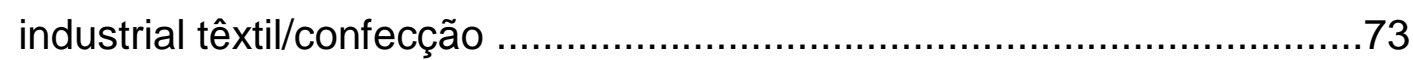

3.3. Considerações sobre a inovação no campo da moda ........................80

3.4. Considerações sobre a inovação no campo do design ........................83

3.5. Gestão do conhecimento e gestão integrada da inovação ..................86

3.6. Classificação das empresas quanto à inovação .................................89

3.7. Características das empresas da cadeia têxtil/confecção

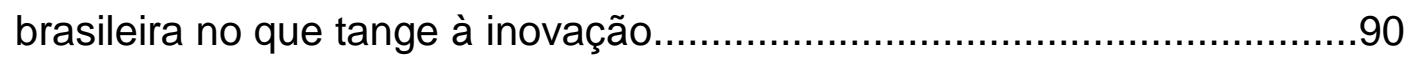

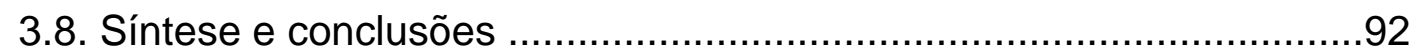

4. Política de promoção e incentivo ao design............................................95

4.1. Panorama internacional das políticas de design.................................96

4.1.1. A mudança de enfoque.......................................................... 96

4.1.2. Estratégias predominantes adotadas nas políticas de design mundial: políticas de suporte, promoção e educação................................100

4.1.3. Políticas adotadas em países selecionados...................................104

4.2. Panorama nacional das políticas de design .....................................114

4.3. A política de design no setor têxtil catarinense................................121

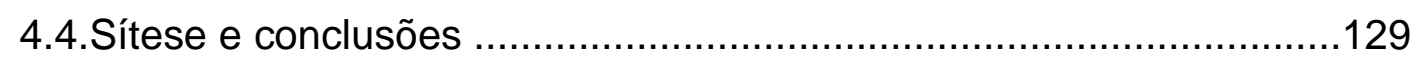

5. Cooperação universidade-empresa (U-E) como instrumento

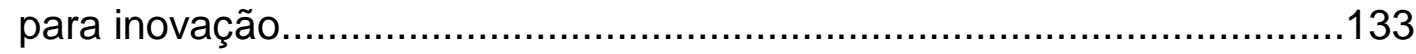

5.1. Especificidade da universidade e da empresa e a evolução da cooperação.

5.2. Referencial teórico das relações universidade

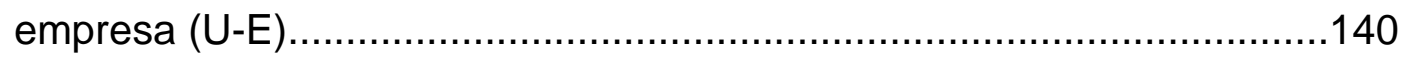

5.2.1. Contribuições da área da psicossociologia .................................143

5.2.2. Contribuições da área da teoria das organizações .......................146

5.3. A criação, transferência e disseminação do conhecimento ..............150

5.4. Financiamento e proteção da inovação ……………........................152

5.5. Síntese e conclusões ..................................................................153

6. Procedimentos metodológicos, método e técnicas de pesquisa ..........156

6.1. caracterização e tipo de pesquisa ................................................156 


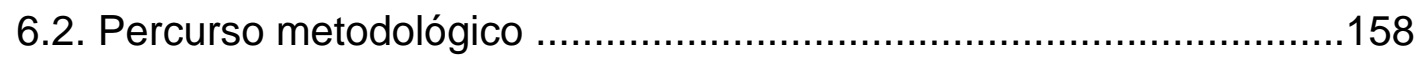

6.3. Primeira aproximação: definição do projeto de pesquisa ...................160

6.3.1 Etapa 1- Definição do objeto de pesquisa .....................................160

6.3.2 Etapa 2- Obtenção de informações de experiência entre U- E........162

6.4 Segunda Aproximação: Pesquisa de Campo......................................162

6.4.1 Etapa 3- Pesquisa com as empresas...........................................163

6.4.2 Etapa 4- Pesquisa com as instituições de ensino ..........................168

6.5 Terceira Aproximação:Pesquisa Qualitativa - opinião dos agentes

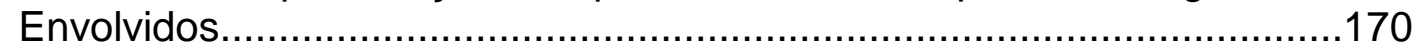

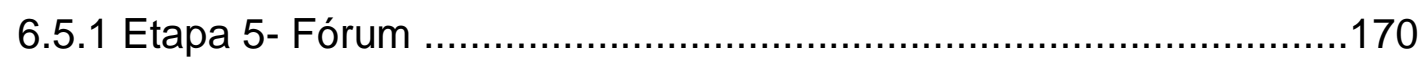

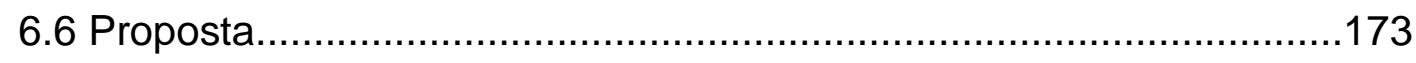

7 Apresentação e análise dos dados da pesquisa .................................174

7.1 Resultados da pesquisa junto às empresas ....................................174

7.1.1. Bloco A: perfil das empresas ..................................................174

7.1.2. Bloco B: demanda de inovação da indústria têxtil

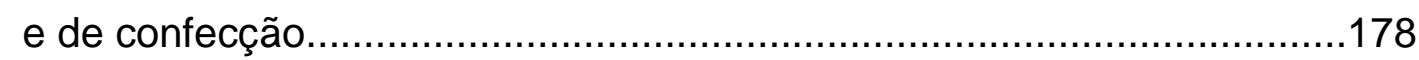

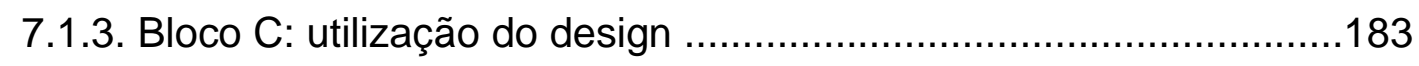

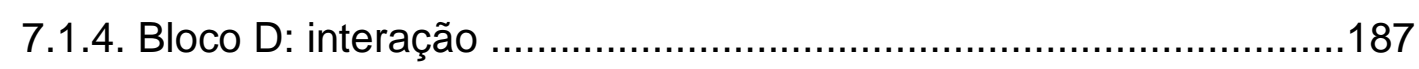

7.2 Resultados da pesquisa junto às Instituições de Ensino .................. 194

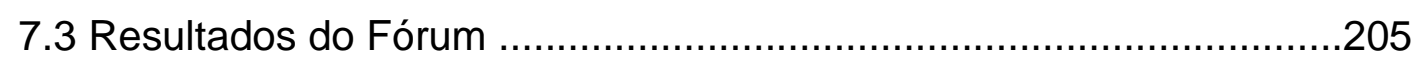

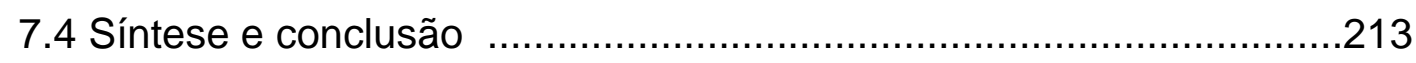

8 Referenciais teórico/operacionais para a política de design do setor têxtil /confecção de moda Catarinense ..................................217

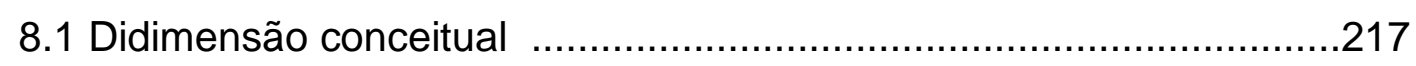

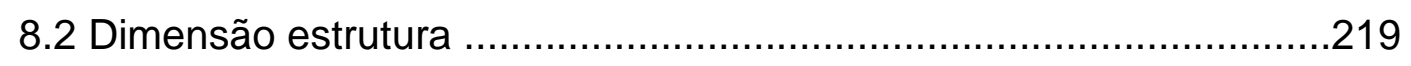

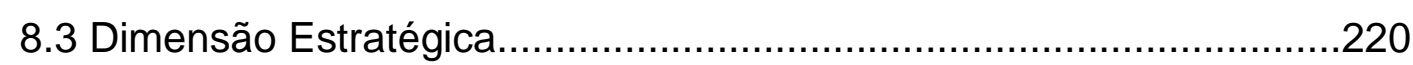

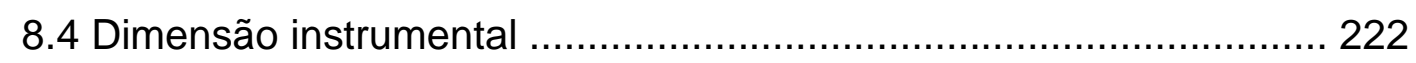

8.5 Síntese e conclusão ...................................................................223

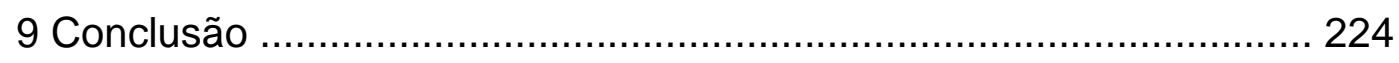


10 Referências bibliográficas

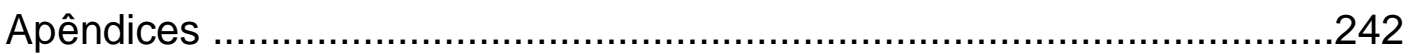

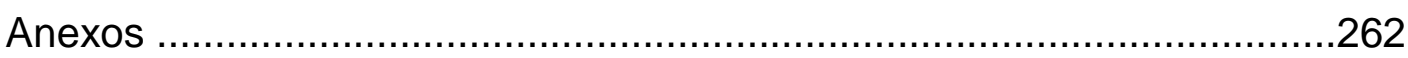

\section{Lista de figuras}

Figura 1.1. Percurso metodológico da pesquisa 25

Figura 2.1. Estrutura da Cadeia Têxtil 33

Figura 2.2. Cadeia de Valor Têxtil/Confecção de Moda 36

Figura 3.1. Tecido xadrez da RenauxView em modelo conceitual $\quad 68$

Figura 3.2. Modelo conceitual UDESC/Iria - 4ª edição do SCMC 69

Figura 3.3. Modelo de Inovação Linear $\quad 77$

Figura 3.4. Modelo de Inovação do Design Centrado no Usuário 78

Figura 3.5. Visão geral da metodologia NUGIN 78

Figura 4.1. Políticas de design $\quad 92$

Figura 4.2. Níveis estratégicos das políticas de design 94

Figura 4.3. Infraestrutura da política de design do Brasil 108

Figura 4.4. Programa SCMC 117

Figura 5.1. Triângulo de Sábato 126

Figura 5.2. Evolução dos Sistemas Nacionais de Inovação 126

Figura 5.3. Estrutura teórica para estudo das elações universidadeempresa 133

Figura 5.4: Hélice Tríplice no Modo 1de Produção do Conhecimento 146

Figura 6.1. Etapas do Percurso Metodológico da Pesquisa 150

Figura 6.2. Mapa de Regionalização de Santa Catarina 156

Figura 7.1. Setor industrial dos principais produtos 166

Figura 7.2. Porte da empresa $\quad 167$

$\begin{array}{ll}\text { Figura 7.3. Âmbito de atuação das empresas } & 167\end{array}$ 
Figura 7.4. Origem do capital da empresa

Figura 7.5: Sustentablidade da estratégia da empresa 169

Figura 7.6. Estratégia da empresa na venda do principal produto $\quad 170$

Figura 7.7. Realização e tipos de atividades inovativas

Figura 7.8. Origem dos recursos para investimento em atividades inovativas

Figura 7.9. Principais áreas para investimento em pesquisa e inovação

Figura 7.10. Resultados esperados com a realização de atividades inovativas ou a implementação da inovação

Figura 7.11. Principais obstáculos à inovação 176

Figura 7.12. Principal função do design na empresa 179

Figura 7.13. Principal fonte de atuação do design 180

Figura 7.14. Responsável pela concepção dos produtos 181

Figura 7.15. Surgimento de ideias para novos produtos 182

Figura 7.16. Envolvimento da empresa em arranjos cooperativos 183

Figura 7.17. Comparativo de opinião U-E sobre principal motivo que leva as empresas a buscar parcerias com as Instituições de Ensino

Figura 7.18. Comparativo de opinião U-E sobre principal motivo que leva Instituições de Ensino a buscarem parcerias com as empresas Figura 7.19. Tipos de instrumentos utilizados na parceria/ cooperação U-E mencionados pelas empresas

Figura 7.20. Participação da empresa no $\operatorname{SCMC}(\mathrm{A})$ e o ano de início (B)

Figura 7.21. Intenção da empresa em permanecer participando ou não no projeto SCMC

Figura 7.22. Relação positiva entre Universidade e empresa nos anos de participação no projeto SCMC

Figura 7.23. Contribuição das Universidades para as empresas nos anos de participação no projeto SCMC

Figura 7.24. Caracterização dos cursos de moda ou design de moda das Instituições de Ensino 
Figura 7.25. Tipos de parcerias que as mantêm com empresas da área têxtil/confecção, excetuando-se a participação no SCMC

Figura 7.26. Existência de sistemas de informação nas Instituições de Ensino (IE) que permitem avaliar as necessidades da Indústria Têxtil e de Confecção de SC

Figura 7.27. Desenvolvimento e apresentação de descrição de projetos inovadores voltado ao sector têxtil/confecção nas Instituições de Ensino

Figura 7.28. Tipos de instrumentos utilizados na parceria/ cooperação U-E mencionados pelas Instituições de Ensino

Figura 7.29. Participação ativa da Instituição de Ensino no processo de desenvolvimento econômico por meio de ações em parceria com as empresas

Figura 7.30. Presença de resistência na Instituição de Ensino com relação ao processo de desenvolvimento econômico por meio de ações em parceria com as empresas

Figura 7.31. Participação/ano de início das Instituições de Ensino no SCMC

Figura 7.32. Atendimento das expectativas das instituições de ensino na relação U-E nos anos de participação no projeto SCMC

Figura 7.33. Tipo de conhecimento adicionado à Instituição de Ensino pela relação com a empresa durante a participação no projeto SCMC

Figura 7.34. Campos de Identificação de Atributos

Figura 7.35. Campos de Identificação de Atributos X Definição de Estratégia

Figura 8.1. Modelo de Gestão de Integração Universidade-Empresa para Política de Design para o Fomento da Inovação 


\section{Lista de quadros}

Quadro 2.1. Elementos principais das configurações nacionais típicas na cadeia têxtil e de confecção

Quadro 2.2. Competitividade da Cadeia Têxtil e de Vestuário Brasileira

Quadro 3.1. Tipos de inovação da indústria têxtil 71

Quadro 4.1. Evolução histórica das políticas e programas de design 89

Quadro 4.2. Ranking Mundial de competitividade do design 2002, 2005 e 2007.

Quadro 5.1. Tipos de Relações na Cooperação UniversidadeEmpresa

Quadro 5.2. Atritos e soluções na transferência do conhecimento

Quadro 6.1. Etapas e Operações Metodológicas da 1ª Aproximação

Quadro 6.2. Etapas e Operações Metodológicas da 2 ${ }^{\mathrm{a}}$ Aproximação 154

Quadro 6.3: Categorias de analise da pesquisa com as empresas $\quad 158$

Quadro 6.4. Etapas e Operações Metodológicas da $3^{\text {a }}$ Aproximação 161

Quadro 6.5.Exemplo de ficha para grupo de trabalho do fórum $\quad 163$

Quadro 7.1: Ficha Grupo de Trabalho - Inovação 208

Quadro 7.2: Ficha Grupo de Trabalho - Design 209

Quadro 7.3: Ficha Grupo de Trabalho - Relação Universidade- 


\section{Lista de tabelas}

Tabela 3.1. Taxas de inovação e incidência sobre a receita líquida de vendas dos dispêndios realizados em atividades inovativas e internas de P\&D na indústria têxtil e de confecção - Brasil, período 2003-2005 e período 2006-2008

Tabela 7.1. Posição do principal produto no mercado 169

Tabela 7.2. Tipo e abrangência de inovação 171

Tabela 7.3. Processo de gestão do conhecimento na empresa 


\section{Lista de siglas}

\begin{tabular}{|l|l|}
\hline APEX & Agência de Promoção de Exportação do Brasil \\
\hline APIs & Arranjos Produtivos Locais \\
\hline ABEPRO & Associação Brasileira de Engenharia de Produto \\
\hline Adegraf & Associação Brasileira dos Designers Gráficos do Distrito Federal \\
\hline ABIT & Associação Brasileira da Indústria Têxtil e de Confecção \\
\hline ABIPTI & Associação Brasileira das Instituições de Pesquisa Tecnológica \\
\hline ABRE & Associação Brasileira de Embalagem \\
\hline Abedesign & Associação Brasileira de Ergonomia \\
\hline Abraweb & Associação Brasileira de Webdesigners e Webmasters \\
\hline ADP & Associação de Designers de Produto \\
\hline ADG & Associação de Designers Gráficos do Brasil \\
\hline APD & Associação de Profissionais de design de Pernambuco \\
\hline APDesign & Associação de Profissionais de Design do Rio Grande do Sul \\
\hline ANPEI & $\begin{array}{l}\text { Associação Nacional de Pesquisa, Desenvolvimento e } \\
\text { Engenharia das das Empresas Inovadoras }\end{array}$ \\
\hline BNDES & Banco Nacional de Desenvolvimento Econômico e Social \\
\hline BRDE & Banco Regional de Desenvolvimento do Extremo Sul \\
\hline CVTCM & Cadeia de Valor Têxtil e de Confecção de Moda \\
\hline Cetiqt & Centro de Tecnologia da Indústria Química e Têxtil \\
\hline
\end{tabular}




\begin{tabular}{|l|l|}
\hline C2i & Centro Internacional de Inovação \\
\hline CEPAL & Comissão Econômica para a América Latina e Caribe \\
\hline CAM & Computer - Aided Manufacturing \\
\hline CAD & Computer - Aided designer \\
\hline CE & Comunidade Europeia \\
\hline CNI & Confederação Nacional da Indústria \\
\hline CNPq & Conselho nacional de desenvolvimento Científico e Tecnológico \\
\hline CIFRE & Convention Industrielle de Formation par la Recherche \\
\hline DMI & European Union Design Management Institute \\
\hline ESDI & Escola Superior de Desenho Industrial \\
\hline EURATEX & European Apparel and Textile Organization \\
\hline FIESC & Federação das Indústrias do Estado de Santa Catarina \\
\hline FIESP & Federação das Indústrias do Estado de São Paulo \\
\hline FIEP & Federação das Indústrias do Estado do paraná \\
\hline FINEP & Financiadora de Estudos e Projetos \\
\hline SITRA & Fundo Nacional Filandês de Pesquisa e Desenvolvimento \\
\hline GCC & Global Commodity Chain \\
\hline GCC & Global Value Chain \\
\hline GE & Grande Empresa \\
\hline IDSA & Industrial Designers's Society of America \\
\hline IBGE & Instituto Brasileiro de Geografia e Estatística \\
\hline KIDP & Instituto Coreano de Promoção do Design \\
\hline ID & Instituto de Design \\
\hline IEMI & Instituto de Estudo e Marketing Industrial \\
\hline TECPAR & Instituto do Paraná \\
\hline IEL & Instituto Evaldo Lodi \\
\hline NID & Instituto Nacional de Design Indiano \\
\hline INT & Instituto Nacional de Tecnologia \\
\hline ICOGRAD & Internatinal Concil of Grafic Design Associations \\
A & International Federations of Interior Architects / Designers \\
\hline ICSID & Internatinal Concil of Societies of Industrial Design \\
\hline IFI & \\
\hline MDE & Média Empresa \\
\hline
\end{tabular}




\begin{tabular}{|l|l|}
\hline ME & Micro Empresa \\
\hline ICT & Ministério da Ciência e Tecnologia \\
\hline MDIC & Ministério do Desenvolvimento, Indústria e Comércio \\
\hline MBC & Movimento Brasileiro Competitivo \\
\hline NZIER & New Zealand Institute of Economic Research \\
\hline NAD & Núcleo de Apoio ao Design ( Senai) \\
\hline NUGIN & Núcleo de Apoio ao Planejamento Gestão da Inovação \\
\hline NID-CT & Núcleo de Inovação e Design - Cadeia Têxtil \\
\hline OCDE & Organização para Cooperação e Desenvolvimento Econômico \\
\hline PE & Pequena Empresa \\
\hline PMEs & Pequenas e Médias Empresas \\
\hline P\&D & Pesquisa e Desenvolvimento \\
\hline PINTEC & Pesquisa Industrial de Inovação Tecnológica \\
\hline e-LAC & Plano de Ação Regional Sobre a Sociedade da Informação na \\
& América Latina e Caribe \\
\hline PUC-Rio & Pontifícia Universidade Católica do Rio de Janeiro \\
\hline PBD & Programa Brasileiro de Designer \\
\hline PAPPE & Programa de Apoio à Pesquisa em Empresas \\
\hline RFDI & $\begin{array}{l}\text { Radio-Frequency IDentification (Identificação por Rádio } \\
\text { Frequência) }\end{array}$ \\
\hline U-E & Relação entre Universidade e Empresa \\
\hline SC & Santa Catarina \\
\hline SCMC & Santa Catarina Moda Contemporânea \\
\hline SENAI & Serviço Nacional de aprendizagem Industria \\
\hline SENAC & Serviço nacional do Comércio \\
\hline TI & Tecnologia da Informação \\
\hline TIC & Tecnologia de Informação e Comunicação \\
\hline UDESC & Universidade do Estado de Santa Catarina \\
\hline UNICAMP & Universidade Estadual de Campinas \\
\hline Unesp & Universidade Estadual Paulista \\
\hline UFPE & Universidade Federal de Pernambuco \\
\hline World Economic Forum's Global Competitiviness Repports \\
\hline
\end{tabular}




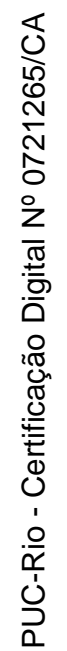

